\title{
Revisiting the generalized Łoś-Tarski theorem
}

\author{
Abhisekh Sankaran \\ Department of Computer Science and Technology, \\ University of Cambridge
}

\begin{abstract}
We present a new proof of the generalized Łoś-Tarski theorem (GLT $(k))$ introduced in [6], over arbitrary structures. Instead of using $\lambda$-saturation as in [6], we construct just the "required saturation" directly using ascending chains of structures. We also strengthen the failure of $\operatorname{GLT}(k)$ in the finite shown in [7], by strengthening the failure of the Loś-Tarski theorem in this context. In particular, we prove that not just universal sentences, but for each fixed $k$, even $\Sigma_{2}^{0}$ sentences containing $k$ existential quantifiers fail to capture hereditariness in the finite. We conclude with two problems as future directions, concerning the Łoś-Tarski theorem and GLT $(k)$, both in the context of all finite structures.
\end{abstract}

\section{INTRODUCTION}

Preservation theorems are a class of results from classical model theory that provide syntactic characterizations of first order (FO) definable classes of arbitrary structures (structures that could be finite or infinite), that are closed under given model-theoretic operations. One of the earliest such results is the Loś-Tarski theorem that states that a class of arbitrary structures defined by an FO sentence is hereditary (closed under substructures) if, and only if, it is definable by a universal sentence (an FO sentence that contains only universal quantifiers) [2]. The theorem in "dual" form characterizes extension closed FO definable classes of arbitrary structures in terms of existential sentences. The theorem extends to theories (sets of sentences) as well. The Eoś-Tarski theorem is historically important for classical model theory since its proof constituted the earliest applications of the FO Compactness theorem (a central result of model theory), and since it triggered off an extensive study of preservation theorems for various other model-theoretic operations (homomorphisms, unions of chains, direct products, etc.), also for logics beyond FO (such as infinitary logics) [3].

Recently [6], a generalization of the Łoś-Tarski theorem was proven by introducing and characterizing a new semantic property that generalizes hereditariness in a parameterized manner. We refer to this property, called preservation under substructures modulo $k$-cruxes in [6], as $k$-hereditariness in this paper. A class of structures is said to be $k$-hereditary if every structure in the class contains a set of at most $k$ elements, called a $k$-crux of the structure, such that all substructures (of the mentioned structure) containing the $k$-crux are also in the class. For instance, consider the class of arbitrary graphs that contain a dominating set of size at most $k$. (A dominating set $S$ in graph is a set of vertices such 
that every vertex in the graph is either in $S$ or adjacent to a vertex in $S$.) This class can be described by the FO sentence $\varphi:=\exists x_{1} \ldots \exists x_{k} \forall y\left(\bigvee_{i=1}^{i=k}\left(\left(y=x_{i}\right) \vee E\left(y, x_{i}\right)\right)\right)$. In any model of $\varphi$, any witnesses to the existential quantifiers of $\varphi$ form a dominating set, and any such set is a $k$-crux of the model; then $\varphi$ defines a $k$-hereditary class. Observe that $\varphi$ is an $\exists^{k} \forall^{*}$ sentence, i.e. a sentence in prenex normal form whose quantifier prefix is a string of $k$ existential quantifiers followed by universal quantifiers 1 . By a similar reasoning as above, it can be shown that any $\exists^{k} \forall^{*}$ sentence defines a $k$-hereditary class. The authors of [6] proved that the converse is true as well, that any FO definable $k$-hereditary class of arbitrary structures is always definable by an $\exists^{k} \forall^{*}$ sentence, thus proving a generalized Łoś-Tarski theorem, that we denote GLT(k) (following [6]). Observe that the Loś-Tarski theorem is a special case of $\operatorname{GLT}(k)$ when $k$ is 0 .

The proof of $\operatorname{GLT}(k)$ from [6] goes via first showing $\operatorname{GLT}(k)$ over a special class of structures called $\lambda$-saturated structures where $\lambda$ is an infinite cardinal. These structures, intuitively speaking, realize many types (maximal consistent sets of formulae in a given number of free variables); in particular, such a structure $\mathfrak{A}$ realizes all the types that are realized in all structures elementarily equivalent to $\mathfrak{A}$, i.e. structures which satisfy the same FO sentences as $\mathfrak{A}$. Then using the fact that every structure has an elementarily equivalent extension that is $\lambda$-saturated for some $\lambda$, the truth of $\operatorname{GLT}(k)$ is "transferred" to all structures. To show GLT $(k)$ over $\lambda$-saturated structures, a notion dual to $k$-hereditariness is introduced, called preservation under $k$-ary covered extensions, that we call $k$-extension closure in this paper. Given a structure $\mathfrak{A}$, define a set $\mathcal{R}$ of substructures of $\mathfrak{A}$ to be a $k$-ary cover of $\mathfrak{A}$ if every set of $k$ elements of $\mathfrak{A}$ is contained in some structure of $\mathcal{R}$. We then say $\mathfrak{A}$ is a $k$-extension of $\mathcal{R}$. A class is $k$-extension closed if every $k$-extension of a set of structures of the class, is also in the class. One sees that a class is $k$-extension closed if, and only if, its complement is $k$-hereditary. Then $\operatorname{GLT}(k)$ is shown by proving its dual form that characterizes $k$-extension closure in terms of $\forall^{k} \exists^{*}$ sentences. The heart of this proof - Lemma 4.2 of [6] - shows that if $\Gamma$ is the theory of the $\forall^{k} \exists^{*}$ implications of a sentence $\varphi$ that defines a $k$-extension closed class, then every $\lambda$-saturated model of $\Gamma$ has a $k$-ary cover consisting of the models of $\varphi$. It follows that the $\lambda$-saturated model then itself models $\varphi$, showing that $\varphi$ and $\Gamma$ are equivalent; then one application of Compactness theorem shows $\varphi$ to be equivalent to a single sentence of $\Gamma$.

The first result of this paper is motivated by the above proof of [6]. In particular, we give a new proof of $\operatorname{GLT}(k)$ that completely avoids using $\lambda$-saturated structures, by making the key observation that the full power of $\lambda$-saturation is hardly used in the proof of the mentioned Lemma 4.2 of [6]. The formulae that play a central role in the proof are not arbitrary FO formulae, but are in fact formulae that have only one quantifier alternation at best. We therefore construct just the "required saturation" as is needed for our proof, by showing a "weaker" version of the mentioned Lemma 4.2, that states that for $\Gamma$ and $\varphi$ as above, every model of $\Gamma$ has an elementarily equivalent extension that might not be $\lambda$-saturated for any $\lambda$, but still contains a $k$-ary cover consisting of models of $\varphi$; see (11) $\rightarrow$ (3) of Lemma 3.5 of this paper. Then showing (the dual form of) GLT $(k)$ over the class of the mentioned elementary extensions is sufficient to transfer GLT $(k)$ out to all structures. The aforementioned implication is in turn shown by defining in the natural way, the more general notion of a $k$-ary cover of a structure in a superstructure of it, and

\footnotetext{
${ }^{1}$ See [5] for a variety of graph properties of interest in parameterized algorithms and finite model theory, that are $k$-hereditary and expressible as $\exists^{k} \forall^{*}$ sentences.
} 
then using (transfinite) induction over the $k$-tuples of elements of a given model $\mathfrak{A}$ of $\Gamma$, to construct an elementary extension $\mathfrak{A}^{\prime}$ of $\mathfrak{A}$ such that $\mathfrak{A}$ has a $k$-ary cover consisting of models of $\varphi$ in $\mathfrak{A}^{\prime}$; see (1) $\rightarrow$ (2) of Lemma 3.5. Applying this implication iteratively to the elementary extensions it gives, we get a chain of structures whose union is an elementary extension of $\mathfrak{A}$ that has a (self-contained) $k$-ary cover of models of $\varphi$; see (2) $\rightarrow$ (3) of Lemma 3.5. Our new proof is therefore much "from the scratch" as opposed to the proof in [6] which uses established notions of model theory.

The second result of this paper is a strengthening of the failure of the Eoś-Tarski theorem in the finite. In the research programme of investigating classical model theoretic results over all finite structures, that was actively pursued from the mid 80s to mid 90s in the context of finite model theory [1], one of the first results identified to fail was the EośTarski theorem. (In fact, Tait had already shown this failure of the Łoś-Tarski theorem in 1959 [9].) Specifically, there is an FO sentence that is hereditary over the class of all finite structures, but that is not equivalent over this class to any universal sentence. In the spirit of [1], one can ask if there is a different syntactic characterization of hereditariness in the finite, or even a syntactic (proper) subfragment of FO that is expressive enough to contain (upto equivalence) all FO sentences that are hereditary when restricted to the finite. We show in Theorem 4.1 that for no fixed $k$, is the class of $\exists^{k} \forall^{*}$ sentences such a subfragment: there is a sentence $\varphi_{k}$ whose models (even including infinite ones) form a hereditary class, and yet $\varphi_{k}$ is not equivalent over all finite structures to any $\exists^{k} \forall^{*}$ sentence.

This result also strengthens the failure of $\operatorname{GLT}(k)$ in the finite as shown in [7]. For every

$k$, the authors of [7] constructed a counterexample to $\operatorname{GLT}(k)$ (over all finite structures) that is $k$-hereditary but not $(k-1)$-hereditary. The sentence $\varphi_{k}$ given by our Theorem 4.1 provides a counterexample to $\operatorname{GLT}(k)$, that is $l$-hereditary for all $l$. The proof of Theorem 4.1 is based on the Ehrenfeucht-Fräissé (EF) method for showing inexpressibility results in FO. We construct for each $n$, a model $\mathfrak{A}_{n}$ and a non-model $\mathfrak{B}_{n}$ of $\varphi_{k}$, such that every $\exists^{k} \forall^{*}$ sentence that is true in $\mathfrak{A}_{n}$ is also true in $\mathfrak{B}_{n}$. The latter is shown by essentially showing that the Duplicator has a winning strategy in a version of the EF game adapted to $\exists^{k} \forall^{*}$ sentences, in which the Spoiler picks up $k$ elements from $\mathfrak{A}_{n}$ in the first move, and $n$ elements from $\mathfrak{B}_{n}$ in the next move. Interestingly, the sentence $\varphi_{k}$ itself turns out to be equivalent to an $\exists^{k+1} \forall^{*}$ sentence.

Paper Organization: In Section 2, we introduce terminology and notation used in the paper, and formally state GLT $(k)$. In Section 3, we present our new proof of GLT $(k)$ and in Section 4, we prove the strengthened failure of the Łoś-Tarski theorem in the finite. We conclude in Section 5 by presenting two problems for future investigation, one concerning the Łoś-Tarski theorem and the other concerning $\operatorname{GLT}(k)$, both in the context of all finite structures.

\section{Preliminaries AND BACKGRound}

We assume the reader is familiar with standard notation and terminology used in the syntax and semantics of FO [2]. A vocabulary $\tau$ is a set of predicate, function and constant symbols. In this paper, we will always be concerned with arbitrary finite vocabularies, unless explicitly stated otherwise. We denote by $\mathrm{FO}(\tau)$ the set of all $\mathrm{FO}$ formulae over vocabulary $\tau$. A sequence $\left(x_{1}, \ldots, x_{k}\right)$ of variables is denoted by $\bar{x}$. A formula $\psi$ whose 
free variables are among $\bar{x}$, is denoted by $\psi(\bar{x})$. A formula with no free variables is called a sentence. An $F O(\tau)$ theory is a set of $\mathrm{FO}(\tau)$ sentences. An $\mathrm{FO}(\tau)$ theory with free variables $\bar{x}$ is a set of $\mathrm{FO}(\tau)$ formulae, all of whose free variables are among $\bar{x}$. When $\tau$ is clear from context, we call an $\mathrm{FO}(\tau)$ theory, a theory simply. We denote by $\mathbb{N}$, the natural numbers including zero. We abbreviate a block of quantifiers of the form $Q x_{1} \ldots Q x_{k}$ by $Q^{k} \bar{x}$ or $Q \bar{x}$ (depending on what is better suited for understanding), where $Q \in\{\forall, \exists\}$ and $k \in \mathbb{N}$. By $Q^{*}$, we mean a block of $k Q$ quantifiers, for some $k \in \mathbb{N}$. For every non-zero $n \in \mathbb{N}$, we denote by $\Sigma_{n}^{0}$ and $\Pi_{n}^{0}$, the classes of all FO sentences in prenex normal form, whose quantifier prefixes begin with $\exists$ and $\forall$ respectively, and consist of $n-1$ alternations of quantifiers. We call $\Sigma_{1}^{0}$ formulae existential and $\Pi_{1}^{0}$ formulae universal. We call $\Sigma_{2}^{0}$ formulae with $k$ existential quantifiers $\exists^{k} \forall^{*}$ formulae, and $\Pi_{2}^{0}$ formulae with $k$ universal quantifiers $\forall^{k} \exists^{*}$ formulae.

We use standard notions of $\tau$-structures (denoted $\mathfrak{A}, \mathfrak{B}$ etc.; we refer to these simply as structures when $\tau$ is clear from context), substructures (denoted $\mathfrak{A} \subseteq \mathfrak{B}$ ), extensions, isomorphisms (denoted $\mathfrak{A} \cong \mathfrak{B}$ ), isomorphic embeddings (denoted $\mathfrak{A} \hookrightarrow \mathfrak{B}$ ), elementary equivalence (denoted $\mathfrak{A} \equiv \mathfrak{B}$ ), elementary substructures (denoted $\mathfrak{A} \preceq \mathfrak{B}$ ) and elementary extensions, as defined in [2]. Given a structure $\mathfrak{A}$, we use $\bigcup_{\mathfrak{A}}$ to denote the universe of $\mathfrak{A}$, and $|\mathfrak{A}|$ to denote the size (or power) of $\mathfrak{A}$ which is the cardinality of $\bigcup_{\mathfrak{A}}$. For an FO sentence $\varphi$ and an FO theory $T$, we denote by $\mathfrak{A} \models \varphi$ and $\mathfrak{A} \models T$ that $\mathfrak{A}$ is a model of $\varphi$ and $T$ respectively. In Section 3 of the paper, we consider structures that could be finite or infinite, whereas in Section 4 we restrict ourselves to only finite structures.

Finally, we use standard abbreviations of English phrases that commonly appear in

mathematical literature. Specifically, 'w.l.o.g' stands for 'without loss of generality', 'iff' stands for 'if and only if', and 'resp.' stands for 'respectively'.

\subsection{The Generalized Łoś-TARsKi THEOREM}

We recall the notions of preservation under substructures modulo $k$-cruxes, $k$-ary covered extensions and preservation under $k$-ary covered extensions introduced in [6], that we resp. call in this paper $k$-hereditariness, $k$-extensions and $k$-extension closure. These notions for $k=0$ correspond exactly to hereditariness, extensions and extension closure resp.

Definition 2.1 (Definition 3.1, [6]).

a. Let $\mathcal{U}$ be a class of arbitrary structures and $k \in \mathbb{N}$. A subclass $\mathcal{S}$ of $\mathcal{U}$ is said to be $k$-hereditary over $\mathcal{U}$, if for every structure $\mathfrak{A}$ of $\mathcal{S}$, there is a set $C \subseteq \cup_{\mathfrak{A}}$ of size $\leq k$ such that if $\mathfrak{B} \subseteq \mathfrak{A}, \mathfrak{B}$ contains $C$ and $\mathfrak{B} \in \mathcal{U}$, then $\mathfrak{B} \in \mathcal{S}$. The set $C$ is called a $k$-crux of $\mathfrak{A}$ w.r.t. $\mathcal{S}$ over $\mathcal{U}$.

b. Given theories $T$ and $V$, we say $T$ is $k$-hereditary modulo $V$, if the class of models of $T \cup V$ is $k$-hereditary over the class of models of $V$. A sentence $\varphi$ is $k$-hereditary modulo $V$ if the theory $\{\varphi\}$ is $k$-hereditary modulo $V$.

Definition 2.2 (Definitions 3.5 and 3.8, [6]).

a. Given a structure $\mathfrak{A}$, a non-empty collection $\mathcal{R}$ of substructures of $\mathfrak{A}$ is said to be a $k$-ary cover of $\mathfrak{A}$ if for every set $C \subseteq \mathrm{U}_{\mathfrak{A}}$ of size $\leq k$, there is a structure in $\mathcal{R}$ that contains $C$. We call $\mathfrak{A}$ a $k$-extension of $\mathcal{R}$.

b. For a class $\mathcal{U}$ of arbitrary structures and $k \in \mathbb{N}$, a subclass $\mathcal{S}$ of $\mathcal{U}$ is said to be $k$-extension closed over $\mathcal{U}$ if for every collection $\mathcal{R}$ of structures of $\mathcal{S}$, if $\mathfrak{A}$ is a $k$ extension of $\mathcal{R}$ and $\mathfrak{A} \in \mathcal{U}$, then $\mathfrak{A} \in \mathcal{S}$. 
c. Given theories $V$ and $T$, we say $T$ is $k$-extension closed modulo $V$ if the class of models of $T \cup V$ is $k$-extension closed over the class of models of $V$. A sentence $\varphi$ is $k$-extension closed modulo $V$ if the theory $\{\varphi\}$ is $k$-extension closed modulo $V$.

We extend the above definitions slightly to formulae and theories with free variables. Given a vocabulary $\tau$, let $\tau_{n}$ denote the vocabulary obtained by expanding $\tau$ with $n$ fresh and distinct constant symbols $c_{1}, \ldots, c_{n}$. For a given $\mathrm{FO}(\tau)$ theory $T\left(x_{1}, \ldots, x_{n}\right)$, let $T^{\prime}$ denote the $\mathrm{FO}\left(\tau_{n}\right)$ theory (without free variables) obtained by substituting $c_{i}$ for $x_{i}$ in $T\left(x_{1}, \ldots, x_{n}\right)$ for each $i \in\{1, \ldots, n\}$. Then we say $T\left(x_{1}, \ldots, x_{n}\right)$ is $k$-hereditary, resp. $k$-extension closed, modulo a theory $V$ if $T^{\prime}$ is $k$-hereditary, resp. $k$-extension closed, modulo $V$ where $V$ is seen as an $\mathrm{FO}\left(\tau_{n}\right)$ theory. A formula $\varphi\left(x_{1}, \ldots, x_{n}\right)$ is $k$-hereditary, resp. $k$-extension closed, modulo $V$ if the theory $\left\{\varphi\left(x_{1}, \ldots, x_{n}\right)\right\}$ is $k$-hereditary, resp. $k$ extension closed, modulo $V$. The following lemma establishes the duality of the introduced preservation properties.

Lemma 2.3 (Lemma 3.9, [6]). Let $\mathcal{U}$ be a class of arbitrary structures, $\mathcal{S}$ be a subclass of $\mathcal{U}$ and $\overline{\mathcal{S}}$ be the complement of $\mathcal{S}$ in $\mathcal{U}$. Then $\mathcal{S}$ is $k$-hereditary over $\mathcal{U}$ iff $\overline{\mathcal{S}}$ is k-extension closed over $\mathcal{U}$, for each $k \in \mathbb{N}$. In particular, if $\mathcal{U}$ is defined by a theory $V$, then a formula $\varphi(\bar{x})$ is k-hereditary modulo $V$ iff $\neg \varphi(\bar{x})$ is k-extension closed modulo $V$.

We now recall GLT $(k)$ as proved in [6]. This theorem gives syntactic characterizations of FO definable $k$-hereditary and $k$-extension closed classes of structures. Observe that the case of $k=0$ gives exactly the Łoś-Tarski theorem. Below, for $\operatorname{FO}(\tau)$ formulae $\varphi(\bar{x})$ and $\psi(\bar{x})$ where $\bar{x}=\left(x_{1}, \ldots, x_{n}\right)$, we say $\varphi(\bar{x})$ is equivalent to $\psi(\bar{x})$ modulo $V$ if for every $\tau$-structure $\mathfrak{A}$ and every $n$-tuple $\bar{a}$ from $\mathfrak{A}$, we have $(\mathfrak{A}, \bar{a})$ is a model of $\{\varphi(\bar{x})\} \cup V$ iff it is a model of $\{\psi(\bar{x})\} \cup V$.

Theorem 2.4 (Generalized Łoś-Tarski theorem: GLT $(k)$; Corollaries 4.4 and 4.6, [6]). Let $\varphi(\bar{x})$ and $V$ be a given formula and theory respectively, and $k \in \mathbb{N}$. Then the following are true:

1. The formula $\varphi(\bar{x})$ is $k$-hereditary modulo $V$ iff it is equivalent modulo $V$ to an $\exists^{k} \forall^{*}$ formula whose free variables are among $\bar{x}$.

2. The formula $\varphi(\bar{x})$ is $k$-extension closed modulo $V$ iff it is equivalent modulo $V$ to a $\forall^{k} \exists^{*}$ formula whose free variables are among $\bar{x}$.

\section{A NEW PROOF OF GLT $(k)$}

We give a new proof to a more general result than Theorem 2.4, from [6]. This result is a generalization of the "extensional" version of GLT $(k)$ to theories. We extend in the natural way the aforestated notion of equivalence modulo a theory, of formulae, to theories with free variables.

Theorem 3.1. A theory $T(\bar{x})$ is k-extension closed modulo a theory $V$ if, and only if, $T(\bar{x})$ is equivalent modulo $V$ to a theory (consisting) of $\forall^{k} \exists^{*}$ formulae all of whose free variables are among $\bar{x}$.

Using the above result, Theorem 2.4 can be proved as below. 
Proof of Theorem 2.4. We prove part (2) of Theorem 2.4. Part (1) of Theorem 2.4 easily follows from part (2) and Lemma 2.3.

The 'If' direction is straightforward. Let $\varphi(\bar{x})$ be equivalent modulo $V$ to the $\forall^{k} \exists$ * formula $\psi(\bar{x})$. Then the theory $\{\varphi(\bar{x})\}$ is equivalent modulo $V$ to the theory $\{\psi(\bar{x})\}$. Then $\{\varphi(\bar{x})\}$, and hence $\varphi(\bar{x})$, is $k$-extension closed modulo $V$ by Theorem 3.1. For the 'Only if' direction, let $\varphi(\bar{x})$ be $k$-extension closed modulo $V$; then so is the theory $\{\varphi(\bar{x})\}$. By Theorem 3.1, $\{\varphi(\bar{x})\}$ is equivalent to a theory $Z(\bar{x})$ of $\forall^{k} \exists^{*}$ formulae whose free variables are among $\bar{x}$. By Compactness theorem, $\{\varphi(\bar{x})\}$ is equivalent modulo $V$ to a finite subset $Y(\bar{x})$ of $Z(\bar{x})$. Then $\varphi(\bar{x})$ is equivalent modulo $V$ to the conjunction of the formulae of $Y(\bar{x})$. Since any conjunction of $\forall^{k} \exists^{*}$ formulae is equivalent (modulo any theory) to a single $\forall^{k} \exists^{*}$ formula, the result follows.

Towards Theorem 3.1, we first recall some important notions and results from the classical model theory literature [2] that are needed for our proof.

Lemma 3.2 (Corollary 5.4.2, Chapter 5 [2]). Let $\mathfrak{A}$ and $\mathfrak{B}$ be structures such that every existential sentence that is true in $\mathfrak{B}$ is true in $\mathfrak{A}$. Then $\mathfrak{B}$ is isomorphically embeddable in an elementary extension of $\mathfrak{A}$.

Given a cardinal $\lambda$, an ascending chain, or simply a chain, $\left(\mathfrak{A}_{\eta}\right)_{\eta<\lambda}$ of structures is a sequence $\mathfrak{A}_{0}, \mathfrak{A}_{1}, \ldots$ of structures such that $\mathfrak{A}_{0} \subseteq \mathfrak{A}_{1} \subseteq \ldots$. The union of this chain is a structure $\mathfrak{A}$ defined as follows: (i) $\bigcup_{\mathfrak{A}}=\bigcup_{\eta<\lambda} \bigcup_{\mathfrak{A}_{\eta}}$, (ii) $c^{\mathfrak{A}}=c^{\mathfrak{A}_{\eta}}$ for every constant symbol $c \in \tau$ and every $\eta<\lambda$, (iii) $R^{\mathfrak{A}}=\bigcup_{\eta<\lambda} R^{\mathfrak{A}_{\eta}}$ for every relation symbol $R \in \tau$, and (iv) $f^{\mathfrak{A}}=\bigcup_{\eta<\lambda} f^{\mathfrak{A}_{\eta}}$ for every function symbol $f \in \tau$ (here, in taking the union of functions, we view an $n$-ary function as its corresponding $(n+1)$-ary relation). Observe that $\mathfrak{A}$ is well-defined. We denote $\mathfrak{A}$ as $\bigcup_{\eta<\lambda} \mathfrak{A}_{\eta}$. If it is additionally the case that $\mathfrak{A}_{0} \preceq \mathfrak{A}_{1} \preceq \ldots$ above, then we say $\left(\mathfrak{A}_{\eta}\right)_{\eta<\lambda}$ is an elementary chain. We now have the following result.

Theorem 3.3 (Tarski-Vaught elementary chain theorem, Theorem 3.1.9, Chapter 3 [2]). Let $\left(\mathfrak{A}_{\eta}\right)_{\eta<\lambda}$ be an elementary chain of structures. Then $\bigcup_{\eta<\lambda} \mathfrak{A}_{\eta}$ is an elementary extension of $\mathfrak{A}_{\eta}$ for each $\eta<\lambda$.

The key element of our proof of Theorem 3.1 is the notion of a $k$-ary cover of a structure $\mathfrak{A}$ in an extension of $\mathfrak{A}$. Below is the definition. Observe that this notion generalizes the notion of $k$-ary cover seen in Definition 2.2 - the latter corresponds to the notion in Definition [3.4, with $\mathfrak{A}^{+}$being the same as $\mathfrak{A}$.

Definition 3.4. Let $\mathfrak{A}$ be a structure and $\mathfrak{A}^{+}$be an extension of $\mathfrak{A}$. A non-empty collection $\mathcal{R}$ of substructures of $\mathfrak{A}^{+}$is said to be a $k$-ary cover of $\mathfrak{A}$ in $\mathfrak{A}^{+}$if for every $k$-tuple $\bar{a}$ of elements of $\mathfrak{A}$, there exists a structure in $\mathcal{R}$ that contains (the elements of) $\bar{a}$.

The following lemma is at the heart of our proof. It (along with its application in proving Theorem [3.1) shows why "full" $\lambda$-saturation as is used in a similar result (Lemma 4.2) in [6], is not needed for Theorem [3.1. Below, $\Gamma=\{\varphi \mid(V \cup T) \rightarrow$ $\varphi$ where $\varphi$ is a $\forall^{k} \exists^{*}$ sentence $\}$. Also a consistent theory is one that has a model.

Lemma 3.5. Let $V$ and $T$ be consistent theories and $k \in \mathbb{N}$. Let $\Gamma$ be the set of $\forall^{k} \exists^{*}$ consequences of $T$ modulo $V$. Then for every model $\mathfrak{A}$ of $V$, the following are equivalent:

1. $\mathfrak{A}$ is a model of $V \cup \Gamma$. 
2. There exists an elementary extension $\mathfrak{A}^{+}$of $\mathfrak{A}$ and a $k$-ary cover $\mathcal{R}$ of $\mathfrak{A}$ in $\mathfrak{A}^{+}$such that $\mathfrak{B} \models(V \cup T)$ for every $\mathfrak{B} \in \mathcal{R}$.

3. There exists an elementary extension $\mathfrak{A}^{+}$of $\mathfrak{A}$ and a $k$-ary cover $\mathcal{R}$ of $\mathfrak{A}^{+}\left(\right.$in $\left.\mathfrak{A}^{+}\right)$ such that $\mathfrak{B} \models(V \cup T)$ for every $\mathfrak{B} \in \mathcal{R}$.

Using the above lemma, Theorem 3.1 can be proved as follows.

Proof of Theorem 3.1. We prove the theorem for theories without free variables; the proof for theories with free variables follows from definitions.

If: Suppose $T$ is equivalent modulo $V$ to a theory $Z$ of $\forall^{k} \exists^{*}$ sentences. Let $\mathfrak{A} \models V$ and let $\mathcal{R}$ be a $k$-ary cover of $\mathfrak{A}$ consisting of models of $V \cup T$. We show that $\mathfrak{A} \models T$. Consider a sentence $\varphi:=\forall^{k} \bar{x} \psi(\bar{x}) \in Z$ where $\psi(\bar{x})$ is an existential formula. Let $\bar{a}$ be a $k$-tuple from $\mathfrak{A}$. Since $\mathcal{R}$ is a $k$-ary cover of $\mathfrak{A}$, there exists $\mathfrak{B} \in \mathcal{R}$ such that $\mathfrak{B} \subseteq \mathfrak{A}$ and $\mathfrak{B}$ contains $\bar{a}$. Since $\mathfrak{B} \models V \cup T$, we have $\mathfrak{B} \models Z$ (since $Z$ and $T$ are equivalent modulo $V)$; then $\mathfrak{B} \models \varphi$ and hence $(\mathfrak{B}, \bar{a}) \models \psi(\bar{x})$. Since existential formulae are preserved under extensions by Łoś-Tarski theorem, we have $(\mathfrak{A}, \bar{a}) \models \psi(\bar{x})$. Since $\bar{a}$ is an arbitrary $k$-tuple of $\mathfrak{A}$, we have $\mathfrak{A} \models \varphi$. Finally, since $\varphi$ is an arbitrary sentence of $Z$, we have $\mathfrak{A} \models Z$, and hence $\mathfrak{A} \models T$.

Only if: Conversely, suppose $T$ is $k$-extension closed modulo $V$. If $V \cup T$ is unsatisfiable, we are trivially done. Else, let $\Gamma$ be the set of $\forall^{k} \exists^{*}$ consequences of $T$ modulo $V$. Then $(V \cup T) \rightarrow(V \cup \Gamma)$. Conversely, suppose $\mathfrak{A} \models(V \cup \Gamma)$. By Lemma 3.5, there exists an elementary extension $\mathfrak{A}^{+}$of $\mathfrak{A}\left(\right.$ hence $\mathfrak{A}^{+} \models V$ ) for which there is a $k$-ary cover consisting of models of $V \cup T$. Then $\mathfrak{A}^{+} \models T$ since $T$ is $k$-extension closed modulo $V$, whereby $\mathfrak{A}=T$. In other words, $(V \cup \Gamma) \rightarrow(V \cup T)$, so that $T$ is equivalent to $\Gamma$ modulo $V$. Then $\Gamma$ is the desired $\forall^{k} \exists^{*}$ theory.

Towards the proof of Lemma 3.5, we would require the following auxiliary lemma.

Lemma 3.6. Let $V, T$ and $\Gamma$ be as in the statement of Lemma [3.5, and suppose $\mathfrak{A} \models$ $(V \cup \Gamma)$. Given an elementary extension $\mathfrak{A}^{\prime}$ of $\mathfrak{A}$ and a $k$-tuple $\bar{a}$ of $\mathfrak{A}$, there exist an elementary extension $\mathfrak{A}^{\prime \prime}$ of $\mathfrak{A}^{\prime}$ and a substructure $\mathfrak{B}$ of $\mathfrak{A}^{\prime \prime}$ such that (i) $\mathfrak{B}$ contains $\bar{a}$ and (ii) $\mathfrak{B} \models(V \cup T)$.

Proof. Let $\operatorname{tp}_{\Pi, \mathfrak{A}, \bar{a}}(\bar{x})$ denote the $\Pi_{1}^{0}$-type of $\bar{a}$ in $\mathfrak{A}$, that is, the set of all $\Pi_{1}^{0}$ formulae that are true of $\bar{a}$ in $\mathfrak{A}$ (so $|\bar{x}|=|\bar{a}|$ ). Let $Z(\bar{x})$ be the theory given by $Z(\bar{x}):=V \cup T \cup \operatorname{tp}_{\Pi, \mathfrak{A}, \bar{a}}(\bar{x})$. We show below that $Z(\bar{x})$ is satisfiable. Assuming this, it follows that if $(\mathfrak{D}, \bar{d}) \models Z(\bar{x})$, then every existential sentence that is true in $(\mathfrak{D}, \bar{d})$ is also true in $(\mathfrak{A}, \bar{a})$, and hence in $\left(\mathfrak{A}^{\prime}, \bar{a}\right)$. Then by Lemma 3.2 , there is an isomorphic embedding $f$ of $(\mathfrak{D}, \bar{d})$ in an elementary extension $\left(\mathfrak{A}^{\prime \prime}, \bar{a}\right)$ of $\left(\mathfrak{A}^{\prime}, \bar{a}\right)$. If the vocabulary of $\mathfrak{A}$ is $\tau$, then taking $\mathfrak{B}$ to be the $\tau$-reduct of the image of $(\mathfrak{D}, \bar{d})$ under $f$, we see that $\mathfrak{B}$ and $\mathfrak{A}^{\prime \prime}$ are as desired.

We show $Z(\bar{x})$ is satisfiable by contradiction. Suppose $Z(\bar{x})$ is inconsistent; then by Compactness theorem, there is a finite subset of $Z(\bar{x})$ that is inconsistent. Since $\operatorname{tp}_{\Pi, \mathfrak{A}, \bar{a}}(\bar{x})$ is closed under finite conjunctions and since each of $\operatorname{tp}_{\Pi, \mathfrak{A}, \bar{a}}(\bar{x}), V$ and $T$ is consistent, there exists $\psi(\bar{x}) \in \operatorname{tp}_{\Pi, \mathfrak{A}, \bar{a}}(\bar{x})$ such that $V \cup T \cup\{\psi(\bar{x})\}$ is inconsistent. In other words, $(V \cup T) \rightarrow \neg \psi(\bar{x})$. Since $V \cup T$ has no free variables, we have $(V \cup T) \rightarrow \varphi$, where $\varphi:=\forall^{k} \bar{x}$ $\neg \psi(\bar{x})$. Observe that $\neg \psi(\bar{x})$ is equivalent to an existential formula; then $\varphi$ is equivalent to a sentence in $\Gamma$, and hence $\mathfrak{A} \models \varphi$. Then $(\mathfrak{A}, \bar{a}) \models \neg \psi(\bar{x})$, contradicting our inference that $\psi(\bar{x}) \in \operatorname{tp}_{\Pi, \mathfrak{A}, \bar{a}}(\bar{x})$. 
Proof of Lemma 3.5. (3) $\rightarrow$ (1): This implication is established along similar lines as the 'If' direction of Theorem 3.1, Let $\varphi:=\forall^{k} \bar{x} \psi(\bar{x})$ for a $\Sigma_{1}^{0}$ formula $\psi(\bar{x})$, be a sentence of $\Gamma$, and let $\bar{a}$ be a $k$-tuple of $\mathfrak{A}$. Since $\mathcal{R}$ is a $k$-ary cover of $\mathfrak{A}^{+}$, there exists $\mathfrak{B}_{\bar{a}} \in \mathcal{R}$ such that $\mathfrak{B}_{\bar{a}}$ contains $\bar{a}$ and $\mathfrak{B}_{\bar{a}} \models(V \cup T)$. Then $\mathfrak{B}_{\bar{a}} \models \Gamma$ so that $\mathfrak{B}_{\bar{a}} \models \varphi$, and hence $\left(\mathfrak{B}_{\bar{a}}, \bar{a}\right) \models \psi(\bar{x})$. Since $\psi(\bar{x})$ is a $\Sigma_{1}^{0}$ formula and $\mathfrak{B}_{\bar{a}} \subseteq \mathfrak{A}^{+}$, we have $\left(\mathfrak{A}^{+}, \bar{a}\right) \models \psi(\bar{x})$ since existential formulae are preserved under extensions by the Eoś-Tarski theorem. Then $(\mathfrak{A}, \bar{a}) \models \psi(\bar{x})$ since $\mathfrak{A} \preceq \mathfrak{A}^{+}$. Since $\bar{a}$ is an arbitrary $k$-tuple of $\mathfrak{A}$, we have $\mathfrak{A} \models \varphi$.

(1) $\rightarrow$ (2): We have two cases here depending on whether $\mathfrak{A}$ is finite or infinite.

(1) $\mathfrak{A}$ is finite: Given a $k$-tuple $\bar{a}$ of $\mathfrak{A}$, by Lemma 3.6 there exists an elementary extension $\mathfrak{A}^{\prime \prime}$ of $\mathfrak{A}$ and a substructure $\mathfrak{B}_{\bar{a}}$ of $\mathfrak{A}^{\prime \prime}$ such that (i) $\mathfrak{B}_{\bar{a}}$ contains $\bar{a}$ and (ii) $\mathfrak{B}_{\bar{a}} \models$ $(V \cup T)$. Since $\mathfrak{A}$ is finite, and since elementary equivalence is the same as isomorphism over finite structures [2], we have $\mathfrak{A}^{\prime \prime}=\mathfrak{A}$. Then taking $\mathfrak{A}^{+}=\mathfrak{A}$ and $\mathcal{R}=\left\{\mathfrak{B}_{\bar{a}} \mid \bar{a} \in\left(\mathrm{U}_{\mathfrak{A}}\right)^{k}\right\}$, we see that $\mathfrak{A}^{+}$and $\mathcal{R}$ are respectively indeed the desired elementary extension of $\mathfrak{A}$ and $k$-ary cover of $\mathfrak{A}$ in $\mathfrak{A}^{+}$.

(2) $\mathfrak{A}$ is infinite: The proof for this case is along the lines of the proof of the characterization of $\Pi_{2}^{0}$ sentences in terms of the property of preservation under unions of chains (see proof of Theorem 3.2.3 in Chapter 3 of [2]). Let $\lambda$ be the successor cardinal of $|\mathfrak{A}|$ and $\left(\bar{a}_{\kappa}\right)_{\kappa<\lambda}$ be an enumeration of the $k$-tuples of $\mathfrak{A}$. For $\eta \leq \lambda$, given sequences $\left(\mathfrak{E}_{\kappa}\right)_{\kappa<\eta}$ and $\left(\mathfrak{F}_{\kappa}\right)_{\kappa<\eta}$ of structures, we say that $\mathcal{P}\left(\left(\mathfrak{E}_{\kappa}\right)_{\kappa<\eta},\left(\mathfrak{F}_{\kappa}\right)_{\kappa<\eta}\right)$ is true iff $\left(\mathfrak{E}_{\kappa}\right)_{\kappa<\eta}$ is an elementary chain and $\mathfrak{A} \preceq \mathfrak{E}_{0}$, and for each $\kappa<\eta$, we have (i) $\mathfrak{F}_{\kappa} \subseteq \mathfrak{E}_{\kappa}$, (ii) $\mathfrak{F}_{\kappa}$ contains $\bar{a}_{\kappa}$, and (iii) $\mathfrak{F}_{\kappa} \models(V \cup T)$. We show below the existence of sequences $\left(\mathfrak{A}_{\kappa}\right)_{\kappa<\lambda}$ and $\left(\mathfrak{B}_{\kappa}\right)_{\kappa<\lambda}$ of structures such that $\mathcal{P}\left(\left(\mathfrak{A}_{\kappa}\right)_{\kappa<\lambda},\left(\mathfrak{B}_{\kappa}\right)_{\kappa<\lambda}\right)$ is true. Then taking $\mathfrak{A}^{+}=\bigcup_{\kappa<\lambda} \mathfrak{A}_{\kappa}$ and $\mathcal{R}=\left\{\mathfrak{B}_{\kappa} \mid \kappa<\lambda\right\}$, we see by Theorem 3.3 that $\mathfrak{A}^{+}$and $\mathcal{R}$ are respectively indeed the elementary extension of $\mathfrak{A}$ and $k$-ary cover of $\mathfrak{A}$ in $\mathfrak{A}^{+}$as desired.

We construct the sequences $\left(\mathfrak{A}_{\kappa}\right)_{\kappa<\lambda}$ and $\left(\mathfrak{B}_{\kappa}\right)_{\kappa<\lambda}$ by constructing for each positive ordinal $\eta<\lambda$, the partial (initial) sequences $\left(\mathfrak{A}_{\kappa}\right)_{\kappa<\eta}$ and $\left(\mathfrak{B}_{\kappa}\right)_{\kappa<\eta}$ and showing that $\mathcal{P}\left(\left(\mathfrak{A}_{\kappa}\right)_{\kappa<\eta},\left(\mathfrak{B}_{\kappa}\right)_{\kappa<\eta}\right)$ is true. We do this by (transfinite) induction on $\eta$. For the base case of $\eta=1$, we see by Lemma 3.6 that if $\mathfrak{A}^{\prime}=\mathfrak{A}$, then there exists an elementary extension $\mathfrak{A}^{\prime \prime}$ of $\mathfrak{A}$ and a substructure $\mathfrak{B}$ of $\mathfrak{A}^{\prime \prime}$ such that (i) $\mathfrak{B}$ contains $\bar{a}_{0}$ and (ii) $\mathfrak{B} \models(V \cup T)$. Then taking $\mathfrak{A}_{0}=\mathfrak{A}^{\prime \prime}$ and $\mathfrak{B}_{0}=\mathfrak{B}$, we see that $\mathcal{P}\left(\left(\mathfrak{A}_{0}\right),\left(\mathfrak{B}_{0}\right)\right)$ is true. As the induction hypothesis, assume that we have constructed sequences $\left(\mathfrak{A}_{\kappa}\right)_{\kappa<\eta}$ and $\left(\mathfrak{B}_{\kappa}\right)_{\kappa<\eta}$ such that $\mathcal{P}\left(\left(\mathfrak{A}_{\kappa}\right)_{\kappa<\eta},\left(\mathfrak{B}_{\kappa}\right)_{\kappa<\eta}\right)$ is true. Then by Theorem 3.3, the structure $\mathfrak{A}^{\prime}=\bigcup_{\kappa<\eta} \mathfrak{A}_{\kappa}$ is such that $\mathfrak{A}_{\kappa} \preceq \mathfrak{A}^{\prime}$ for each $\kappa<\eta$. Then for the tuple $\bar{a}_{\eta}$ of $\mathfrak{A}$, by Lemma 3.6, there exists an elementary extension $\mathfrak{C}$ of $\mathfrak{A}^{\prime}$ and a substructure $\mathfrak{D}$ of $\mathfrak{C}$ such that (i) $\mathfrak{D}$ contains $\bar{a}_{\eta}$ and (ii) $\mathfrak{D} \models(V \cup T)$. Then taking $\mathfrak{A}_{\eta}=\mathfrak{C}$ and $\mathfrak{B}_{\eta}=\mathfrak{D}$, and letting $\mu$ be the successor ordinal of $\eta$, we see that $\mathcal{P}\left(\left(\mathfrak{A}_{\kappa}\right)_{\kappa<\mu},\left(\mathfrak{B}_{\kappa}\right)_{\kappa<\mu}\right)$ is true, completing the induction.

$(2) \rightarrow(3)$ : Applying (2) to $\mathfrak{A}$ and then iteratively to the elementary extensions that (2) $\overline{\text { produces, }}$ we get a sequence $\left(\mathfrak{A}_{i}\right)_{i \geq 0}$ of structures and a sequence $\left(\mathcal{R}_{i}\right)_{i \geq 0}$ of collections of structures with the following properties:

1. $\left(\mathfrak{A}_{i}\right)_{i \geq 0}$ is an elementary chain such that $\mathfrak{A}_{0}=\mathfrak{A}$ (whereby $\mathfrak{A}_{i} \models V$ for $i \geq 0$ ).

2. For each $i \geq 0, \mathcal{R}_{i}$ is a $k$-ary cover of $\mathfrak{A}_{i}$ in $\mathfrak{A}_{i+1}$ such that $\mathfrak{B} \models(V \cup T)$ for every $\mathfrak{B} \in \mathcal{R}_{i}$.

Consider the structure $\mathfrak{A}^{+}=\bigcup_{i>0} \mathfrak{A}_{i}$. By Theorem 3.3. we have $\mathfrak{A}_{i} \preceq \mathfrak{A}^{+}$for each $i \geq 0$, and (hence) that $\mathfrak{A}^{+} \models V$. Consider any $k$-tuple $\bar{a}$ of $\mathfrak{A}^{+}$; there exists $j \geq 0$ such $\bar{a}$ is contained in $\mathfrak{A}_{j}$. Then there exists a structure $\mathfrak{B}_{\bar{a}} \in \mathcal{R}_{j}$ such that (i) $\mathfrak{B}_{\bar{a}}$ contains $\bar{a}$ 
and (ii) $\mathfrak{B}_{\bar{a}} \models(V \cup T)$. Since $\mathfrak{B}_{\bar{a}} \in \mathcal{R}_{j}$, we have $\mathfrak{B}_{\bar{a}} \subseteq \mathfrak{A}_{j+1}$ and since $\mathfrak{A}_{j+1} \preceq \mathfrak{A}^{+}$, we have $\mathfrak{B}_{\bar{a}} \subseteq \mathfrak{A}^{+}$. Then $\mathcal{R}=\left\{\mathfrak{B}_{\bar{a}} \mid \bar{a}\right.$ is a $k$-tuple from $\left.\mathfrak{A}^{+}\right\}$is the desired $k$-ary cover of $\mathfrak{A}^{+}$ such that $\mathfrak{B} \models(V \cup T)$ for each $\mathfrak{B} \in \mathcal{R}$.

\section{A Stronger FAILURE of Łoś-TARski theorem In THE FINITE}

In this section, we strengthen the known failure of the Łoś-Tarski theorem in the finite [1]. As a consequence, we get a strengthening of the failure of $\mathrm{GLT}(k)$ in the finite for each $k$, over the one proved in [7]. Below, by $\varphi_{k}$ is $(k-)$ hereditary over $\mathcal{S}$ we mean that the class of finite models of $\varphi_{k}$ is $(k$-)hereditary over $\mathcal{S}$.

Theorem 4.1. There exists a vocabulary $\tau$ such that if $\mathcal{S}$ is the class of all finite $\tau$ structures, then for each $k \geq 0$, there exists an $F O(\tau)$ sentence $\varphi_{k}$ that is hereditary over $\mathcal{S}$, but that is not equivalent over $\mathcal{S}$, to any $\exists^{k} \forall^{*}$ sentence. It follows that there is a sentence that is $k$-hereditary over $\mathcal{S}$ ( $\varphi_{k}$ being one such sentence) but that is not equivalent over $\mathcal{S}$ to any $\exists^{k} \forall^{*}$ sentence.

Proof. The second part of the theorem follows from the first part since a sentence that is hereditary over $\mathcal{S}$ is also $k$-hereditary over $\mathcal{S}$ for each $k \geq 0$. We now prove the first part of the theorem. Consider the vocabulary $\tau=\{\leq, S, P, c, d\}$ where $\leq$ and $S$ are binary relation symbols, $P$ is a unary relation symbol, and $c$ and $d$ are constant symbols. The sentence $\varphi_{k}$ is constructed along the lines of the counterxample to the Łoś-Tarski theorem in the finite as given in [1].

$$
\begin{aligned}
& \varphi_{k}:=\left(\xi_{1} \wedge \xi_{2} \wedge \xi_{3}\right) \wedge \neg\left(\xi_{4} \wedge \xi_{5}\right) \\
& \xi_{1}:=\text { " } \leq \text { is a linear order" } \\
& \xi_{2}:=\text { " } \text { is minimum under } \leq \text { and } d \text { is maximum under } \leq " \\
& \xi_{3}:=\forall x \forall y S(x, y) \rightarrow \text { " } y \text { is the successor of } x \text { under } \leq " \\
& \xi_{4}:=\forall x(x \neq d) \rightarrow \exists y S(x, y) \\
& \xi_{5}:=\text { "There exist at most } k \text { elements in (the set interpreting) } P \text { " }
\end{aligned}
$$

Each of $\xi_{1}, \xi_{2}, \xi_{3}$ and $\xi_{5}$ can be expressed using a universal sentence. In particular, $\xi_{1}$ and $\xi_{3}$ can be expressed using a $\forall^{3}$ sentence each, $\xi_{2}$ using a $\forall$ sentence, and $\xi_{5}$ using a $\forall^{k+1}$ sentence. Then $\varphi_{k}$ is equivalent to an $\exists^{k+1} \forall^{3}$ sentence.

We first show that $\varphi_{k}$ is hereditary over $\mathcal{S}$, by showing that $\psi_{k}:=\neg \varphi_{k}$ is extension closed over $\mathcal{S}$. Let $\mathfrak{A} \models \psi_{k}$ and $\mathfrak{A} \subseteq \mathfrak{B}$. If $\alpha:=\left(\xi_{1} \wedge \xi_{2} \wedge \xi_{3}\right)$ is such that $\mathfrak{A} \models \neg \alpha$, then since $\neg \alpha$ is equivalent to an existential sentence, we have $\mathfrak{B} \models \neg \alpha$; then $\mathfrak{B} \models \psi_{k}$. Else, $\mathfrak{A} \models \alpha \wedge \xi_{4}$. Let $b$ be an element of $\mathfrak{B}$ that is not in $\mathfrak{A}$. Then there are two cases, in both which we show that $\mathfrak{B} \models \psi_{k}$, as below:

1. $\left(\mathfrak{B}, a_{1}, b, a_{2}\right) \models((x \leq y) \wedge(y \leq z))$ for two elements $a_{1}, a_{2}$ of $\mathfrak{A}$ such that $\left(\mathfrak{A}, a_{1}, a_{2}\right) \models$ $S(x, z)$; then $\mathfrak{B} \models \neg \xi_{3}$ and hence $\mathfrak{B} \models \psi_{k}$.

2. $(\mathfrak{B}, b) \models((d \leq x) \vee(x \leq c))$. Since the interpretations of $c, d$ in $\mathfrak{B}$ are resp. the same as those of $c, d$ in $\mathfrak{A}$, we have $\mathfrak{B} \models \neg \xi_{2}$ and hence $\mathfrak{B} \models \psi_{k}$.

We now show that $\varphi_{k}$ is not equivalent over $\mathcal{S}$ to any $\exists^{k} \forall^{*}$ sentence. Towards a contradiction, suppose $\varphi_{k}$ is equivalent over $\mathcal{S}$ to the sentence $\gamma:=\exists x_{1} \ldots \exists x_{k} \forall^{n} \bar{y} \beta\left(x_{1}, \ldots, x_{k}, \bar{y}\right)$, where $\beta$ is a quantifier-free formula. Consider the structure $\mathfrak{A}=\left(\cup_{\mathfrak{A}}, \leq^{\mathfrak{A}}, S^{\mathfrak{A}}, P^{\mathfrak{A}}, c^{\mathfrak{A}}, d^{\mathfrak{A}}\right)$, where the universe $\cup_{\mathfrak{A}}=\{1, \ldots,(8 n+1) \times(k+1)\}, \leq^{\mathfrak{A}}$ and $S^{\mathfrak{A}}$ are respectively the 
usual linear order and successor relation on $\cup_{\mathfrak{A}}, c^{\mathfrak{A}}=1, d^{\mathfrak{A}}=(8 n+1) \times(k+1)$ and $P^{\mathfrak{A}}=\{(4 n+1)+i \times(8 n+1) \mid i \in\{0, \ldots, k\}\}$. We see that $\mathfrak{A} \models\left(\xi_{1} \wedge \xi_{2} \wedge \xi_{3} \wedge \xi_{4} \wedge \neg \xi_{5}\right)$ and hence $\mathfrak{A} \models \varphi_{k}$. Then $\mathfrak{A} \models \gamma$. Let $a_{1}, \ldots, a_{k}$ be the witnesses in $\mathfrak{A}$ to the $k$ existential quantifiers of $\gamma$.

It is clear that there exists $i^{*} \in\{0, \ldots, k\}$ such that $a_{j}$ does not belong to $\{(8 n+1) \times$ $\left.i^{*}+1, \ldots,(8 n+1) \times\left(i^{*}+1\right)\right\}$ for each $j \in\{1, \ldots, k\}$. Then consider the structure $\mathfrak{B}$ that is identical to $\mathfrak{A}$ except that $P^{\mathfrak{B}}=P^{\mathfrak{A}} \backslash\left\{(4 n+1)+i^{*} \times(8 n+1)\right\}$. It is clear from the definition of $\mathfrak{B}$ that $\mathfrak{B} \models\left(\xi_{1} \wedge \xi_{2} \wedge \xi_{3} \wedge \xi_{4} \wedge \xi_{5}\right)$ and hence $\mathfrak{B} \models \neg \varphi_{k}$. We now show a contradiction by showing that $\mathfrak{B} \models \gamma$.

We show that $\mathfrak{B} \models \gamma$ by showing that $\left(\mathfrak{B}, a_{1}, \ldots, a_{k}\right) \models \forall^{n} \bar{y} \beta\left(x_{1}, \ldots, x_{k}, \bar{y}\right)$. This is in turn done by showing that for any $n$-tuple $\bar{e}=\left(e_{1}, \ldots, e_{n}\right)$ from $\mathfrak{B}$, there exists an $n$-tuple $\bar{f}=\left(f_{1}, \ldots, f_{n}\right)$ from $\mathfrak{A}$ such that the (partial) map $\rho: \mathfrak{B} \rightarrow \mathfrak{A}$ given by $\rho(1)=1, \rho((8 n+1) \times(k+1))=(8 n+1) \times(k+1), \rho\left(a_{j}\right)=a_{j}$ for $j \in\{1, \ldots, k\}$ and $\rho\left(e_{j}\right)=f_{j}$ for $j \in\{1, \ldots, n\}$ is such that $\rho$ is a partial isomorphism from $\mathfrak{B}$ to $\mathfrak{A}$. Then since $\left(\mathfrak{A}, a_{1}, \ldots, a_{k}\right) \models \forall^{n} \bar{y} \beta\left(x_{1}, \ldots, x_{k}, \bar{y}\right)$, we have $\left(\mathfrak{A}, a_{1}, \ldots, a_{k}, \bar{f}\right) \models \beta\left(x_{1}, \ldots, x_{k}, \bar{y}\right)$ whereby $\left(\mathfrak{B}, a_{1}, \ldots, a_{k}, \bar{e}\right) \models \beta\left(x_{1}, \ldots, x_{k}, \bar{y}\right)$. As $\bar{e}$ is an arbitrary $n$-tuple from $\mathfrak{B}$, we have $\left(\mathfrak{B}, a_{1}, \ldots, a_{k}\right) \models \forall^{n} \bar{y} \beta\left(x_{1}, \ldots, x_{k}, \bar{y}\right)$.

Define a contiguous segment in $\mathfrak{B}$ to be a set of $l$ distinct elements of $\mathfrak{B}$, for some $l \geq 1$, that are contiguous w.r.t. the linear ordering in $\mathfrak{B}$. That is, if $b_{1}, \ldots, b_{l}$ are the distinct elements of the aforesaid contiguous segment, then $\left(b_{j}, b_{j+1}\right) \in S^{\mathfrak{B}}$ for $j \in\{1, \ldots, l-1\}$. We represent such a contiguous segment as $\left[b_{1}, b_{l}\right]$, and view it as an interval in $\mathfrak{B}$. Given an $n$-tuple $\bar{e}$ from $\mathfrak{B}$, a contiguous segment of $\bar{e}$ in $\mathfrak{B}$ is a contiguous segment in $\mathfrak{B}$, all of whose elements belong to (the set underlying) $\bar{e}$. A maximal contiguous segment of $\bar{e}$ in $\mathfrak{B}$ is a contiguous segment of $\bar{e}$ in $\mathfrak{B}$ that is not strictly contained in another contiguous segment of $\bar{e}$ in $\mathfrak{B}$. Let CS be the set of all maximal contiguous segments of $\bar{e}$ in $\mathfrak{B}$. Let $\mathrm{CS}_{1} \subseteq \mathrm{CS}$ be the set of all those segments of CS that have an intersection with the set $\left\{1, \ldots,(8 n+1) \times i^{*}\right\} \cup\left\{(8 n+1) \times\left(i^{*}+1\right)+1, \ldots,(8 n+1) \times(k+1)\right\}$. Let $\mathrm{CS}_{2}=\mathrm{CS} \backslash \mathrm{CS}_{1}$. Then all intervals in $\mathrm{CS}_{2}$ are contained in the interval $\left[(8 n+1) \times i^{*}+1,(8 n+1) \times\left(i^{*}+1\right)\right]$. Let $\mathrm{CS}_{2}=\left\{\left[i_{1}, j_{1}\right],\left[i_{2}, j_{2}\right] \ldots,\left[i_{r}, j_{r}\right]\right\}$ such that $i_{1} \leq j_{1}<i_{2} \leq j_{2}<\ldots<i_{r} \leq j_{r}$. Observe that $r \leq n$. Let $\mathrm{CS}_{3}$ be the set of contiguous segments in $\mathfrak{A}$ defined as $\mathrm{CS}_{3}=$ $\left\{\left[i_{1}^{\prime}, j_{1}^{\prime}\right],\left[i_{2}^{\prime}, j_{2}^{\prime}\right], \ldots,\left[i_{r}^{\prime}, j_{r}^{\prime}\right]\right\}$ where $i_{1}^{\prime}=(8 n+1) \times i^{*}+n+1, j_{1}^{\prime}=i_{1}^{\prime}+\left(j_{1}-i_{1}\right)$, and for $2 \leq l \leq r$, we have $i_{l}^{\prime}=j_{l-1}^{\prime}+2$ and $j_{l}^{\prime}=i_{l}^{\prime}+\left(j_{l}-i_{l}\right)$. Observe that the sum of the lengths of the segments of $\mathrm{CS}_{2}$ is at most $n$, so that $j_{r}^{\prime} \leq(8 n+1) \times i^{*}+3 n+1$.

Now consider the tuple $\bar{f}=\left(f_{1}, \ldots, f_{n}\right)$ defined using $\bar{e}=\left(e_{1}, \ldots, e_{n}\right)$ as follows. Let Elements $\left(\mathrm{CS}_{1}\right)$, resp. Elements $\left(\mathrm{CS}_{2}\right)$, denote the elements contained in the segments of $\mathrm{CS}_{1}$, resp. $\mathrm{CS}_{2}$. For $1 \leq l \leq n$, if $e_{l} \in \operatorname{Elements}\left(\mathrm{CS}_{1}\right)$, then $f_{l}=e_{l}$. Else suppose $e_{l}$ belongs to the segment $\left[i_{s}, j_{s}\right]$ of $\mathrm{CS}_{2}$ where $1 \leq s \leq r$, and suppose that $e_{l}=i_{s}+t$ for some $t \in\left\{0, \ldots,\left(j_{s}-i_{s}\right)\right\}$. Then choose $f_{l}=i_{s}^{\prime}+t$. We now verify that the (partial) map $\rho: \mathfrak{B} \rightarrow \mathfrak{A}$ that is given by $\rho(1)=1, \rho((8 n+1) \times(k+1))=(8 n+1) \times(k+1), \rho\left(a_{j}\right)=a_{j}$ for $j \in\{1, \ldots, k\}$ and $\rho\left(e_{l}\right)=f_{l}$ for $l \in\{1, \ldots, n\}$, is indeed a partial isomorphism from $\mathfrak{B}$ to $\mathfrak{A}$. 


\section{Conclusion And Future Directions}

In this paper, we presented a new proof of the extensional form of the generalized LośTarski theorem $(\mathrm{GLT}(k))$ for theories first shown in [6], and thereby obtained a new proof of the theorem for sentences in both its forms substructural and extensional. Our proof avoids using $\lambda$-saturation as used in [6], and instead constructs structures with just the "needed saturation" to prove the theorem. As our second result, we presented a strengthening of the failure of the Loś-Tarski theorem in the finite by showing that not only universal sentences, but even $\exists^{k} \forall^{*}$ sentences for any fixed $k$ are not expressive enough to capture the semantic property of hereditariness in the finite.

We now mention two future directions concerning our results. The first is in connection with the Eoś-Tarski theorem in the finite. The counterexample to this theorem in the finite as presented in [1] uses two binary relations and two constants. But what happens if the vocabulary contains only one binary relation and some constants/unary relations? There are positive results shown when the binary relation is constrained to be interpreted as special kinds of posets, specifically linear orders or (more generally) poset-theoretic trees, or special kinds of graphs, specifically subclasses of bounded clique-width graphs such as classes of bounded tree-depth/shrub-depth and $m$-partite cographs [4]. (In fact, over all these classes, even $\operatorname{GLT}(k)$ is true for all $k$.) But the case of an unconstrained binary relation remains open, motivating the following question 2

Problem 5.1. Is the (relativized version of the) Eoś-Tarski theorem true over all finite colored directed graphs? The same question also for undirected graphs.

Our second future direction concerns $\operatorname{GLT}(k)$ over all finite structures. Theorem 4.1 exhibits for each $k$, a sentence $\varphi_{k}$ that is hereditary over all finite structures but that is not equivalent over this class to any $\exists^{k} \forall^{*}$ sentence. We however observe that $\varphi_{k}$ is itself equivalent to an $\exists^{k+1} \forall^{*}$ sentence. So that this counterexample to $\operatorname{GLT}(k)$ is not a counterexample to $\mathrm{GLT}(k+1)$. This raises the natural question of whether all counterexamples to $\operatorname{GLT}(k)$ in the finite, are simply $\Sigma_{2}^{0}$ sentences, or sentences equivalent to these. Given that any $\Sigma_{2}^{0}$ sentence is $k$-hereditary for some $k$, we pose the aforesaid question as the following problem.

Problem 5.2. Is it the case that over the class of all finite structures, a sentence is $k$-hereditary for some $k$ if, and only if, it is equivalent to a $\Sigma_{2}^{0}$ sentence?

Observe that the version of Problem 5.2 in which arbitrary structures are considered instead of finite structures, has a positive answer due to Theorem 2.4 (which is a stronger statement). Much like the Łoś-Tarski theorem, results from classical model theory almost invariably fail in the finite [1]. Resolving Problem [5.2 in the affirmative would then give us a preservation theorem that survives passage to all finite structures.

\footnotetext{
${ }^{2}$ We remark that special cases of [5.1 for different fragments of FO were studied in [8] and the Łoś-Tarski theorem was verified to be true for these cases over all finite structures, even for arbitrary finite relational vocabularies.
} 


\section{REFERENCES}

[1] Natasha Alechina and Yuri Gurevich. Syntax vs. semantics on finite structures. In Structures in Logic and Computer Science. A Selection of Essays in Honor of A. Ehrenfeucht, pages 14-33. Springer-Verlag, 1997.

[2] Chen C. Chang and Howard J. Keisler. Model Theory, volume 73. Elsevier, 1990.

[3] Wilfrid Hodges. Model Theory (Draft 20 Jul 00), 2000. http://wilfridhodges.co.uk/history07.pdf.

[4] Abhisekh Sankaran. A Generalization of the Eoś-Tarski Preservation Theorem. PhD thesis, Dept. of Computer Science and Engineering, Indian Institute of Technology Bombay, 2016. Available at: CoRR abs/1609.06297 (2016).

[5] Abhisekh Sankaran. A generalization of the Łoś-Tarski preservation theorem - dissertation summary. CoRR, abs/1811.01014, 2018.

[6] Abhisekh Sankaran, Bharat Adsul, and Supratik Chakraborty. A generalization of the Łoś-Tarski preservation theorem. Ann. Pure Appl. Logic, 167(3):189-210, 2016.

[7] Abhisekh Sankaran, Bharat Adsul, Vivek Madan, Pritish Kamath, and Supratik Chakraborty. Preservation under substructures modulo bounded cores. In Proc. of WoLLIC, pages 291-305, 2012.

[8] Abhisekh Sankaran, Bharat Adsul, Vivek Madan, Pritish Kamath, and Supratik Chakraborty. Preservation under substructures modulo bounded cores. CoRR, abs/1205.1358, 2012.

[9] William W. Tait. A counterexample to a conjecture of Scott and Suppes. J. Symb. Logic, 24(1):15-16, 1959. 